RU-97-78

KUNS-1465 HE(TH) 97/14

\title{
Gauge Freedom in Chiral Gauge Theory with Vacuum Overlap*
}

\author{
Yoshio Kikukawa ${ }^{\text {a }}$ \\ ${ }^{a}$ Department of Physics and Astronomy, Rutgers University, Piscataway, NJ 08855-0849, USA
}

Dynamical nature of the gauge degrees of freedom and its effect to fermion spectrum are studied at $\beta=\infty$ for two- and four-dimensional nonabelian chiral gauge theories in the vacuum overlap formalism. It is argued that the disordered gauge degrees of freedom does not contradict to the chiral spectrum of lattice fermion.

\section{Introduction - Pure gauge limit}

In the vacuum overlap formalism[1] of a generic chiral gauge theory, gauge symmetry is explicitly broken by the complex phase of fermion determinant. In order to restore the gauge invariance, gauge average - the integration along gauge orbit- is invoked. Then, what is required for the dynamical nature of the gauge freedom at $\beta=\infty$ (pure gauge limit) is that the global gauge symmetry is not broken spontaneously and the bosonic fields of the gauge freedom have large mass compared to a typical mass scale of the theory so as to decouple from physical spectrum 2, 1].

However, through the analysis of the waveguide model[3, 4 , it has been claimed that this required disordered nature of the gauge freedom causes the vector-like spectrum of fermion [5]. In this argument, the fermion correlation functions at the waveguide boundaries were examined. One may think of the counter parts of these correlation functions in the overlap formalism by putting creation and annihilation operators in the overlap of vacua with the same signature of mass. Let us refer this kind of correlation function as boundary correlation function and the correlation function in the original definition as overlap correlation function. We should note that the boundary correlation functions are no more the observables in the sense defined in the overlap formalism; they cannot be expressed by the overlap of two vacua with their phases fixed by the Wigner-Brillouin

\footnotetext{
*Talk presented at Lattice '97, Edinburgh.

$\dagger$ On leave of absence from: Department of Physics, Kyoto

University, Kyoto 606-01, Japan
}

phase convention. But, they are still relevant because they can probe the auxiliary fermionic system for the definition of the complex phase of chiral determinant and therefore the anomaly (the Wess-Zumino-Witten term). If massless chiral states could actually appear in the boundary correlation functions, we would have difficulty defining the complex phase.

Our objective in this article is to argue that the disordered nature of the gauge degrees of freedom does not contradict to the chiral spectrum of lattice fermion in the vacuum overlap formalism. For this goal, we consider the pure gauge limit of two- and four-dimensional $S U(N)$ nonablelian chiral gauge theories $[6, \sqrt{6}$. The partition functions of these theories can be written in general as

$$
\begin{aligned}
Z= & \int[d U] \exp \left(-\beta S_{G}\right) \times \\
& \prod_{\text {rep. }}\left(\frac{\langle+\mid v+\rangle}{|\langle+\mid v+\rangle|}\langle v+\mid v-\rangle \frac{\langle v-\mid-\rangle}{|\langle v-\mid-\rangle|}\right) .
\end{aligned}
$$

In this formula[1],$|v+\rangle$ and $|v-\rangle$ are the vacua of the second-quantized Hamiltonians of the threeor five-dimensional Wilson fermion with positive and negative bare masses, respectively. $|+\rangle$ and $|-\rangle$ are corresponding free vacua. The WignerBrillouin phase convention is explicitly implemented by the overlaps of vacua with the same signature of mass. $\prod_{r e p}$. stands for the product over all Weyl fermion multiplets in an anomaly free representation. $S_{G}$ is the gauge action.

At $\beta=\infty$, the gauge link variable is given in the pure gauge form:

$U_{n \mu}=g_{n} g_{n+\mu}^{\dagger} \quad g_{n} \in S U(N)$. 
Then the model describes the gauge degrees of freedom coupled to fermion through the gauge non-invariant piece of the complex phase of chiral determinants.

$$
\begin{aligned}
Z & =\int[d g] \prod_{\text {rep. }}\left(\frac{\langle+|\hat{G}|+\rangle}{|\langle+|\hat{G}|+\rangle|}\langle+\mid-\rangle \frac{\left\langle-\left|\hat{G}^{\dagger}\right|-\right\rangle}{\left|\left\langle-\left|\hat{G}^{\dagger}\right|-\right\rangle\right|}\right) \\
& \equiv \int d \mu[g] .
\end{aligned}
$$

$\hat{G}$ is the operator of the gauge transformation given by:

$\hat{G}=\exp \left(\hat{a}_{n}^{\dagger i}\{\log g\}_{i}{ }^{j} \hat{a}_{n j}\right)$.

In this limit, one of the possible definitions of the boundary correlation functions is given as follows for the case of the negative mass and in the representation $r$ :

$$
\begin{aligned}
\left\langle\phi_{n i} \phi_{m}^{\dagger}{ }^{j}\right\rangle_{-r} \equiv & \frac{1}{Z} \int d \mu[g] \times \\
& \frac{\left\langle-\left|\hat{G}^{\dagger}\left\{\hat{a}_{n i} \hat{a}_{m}^{\dagger}{ }^{j}-\frac{1}{2} \delta_{n m} \delta_{i}^{j}\right\}\right|-\right\rangle_{r}}{\left\langle-\left|\hat{G}^{\dagger}\right|-\right\rangle_{r}} .
\end{aligned}
$$

Note that it transforms under the $S U(N)$ global gauge transformation as

$\left\langle\phi_{n i} \phi_{m}^{\dagger}{ }^{j}\right\rangle_{-r} \longrightarrow\left(g_{0}^{s}\right)\left\langle\phi_{n s} \phi_{m}^{\dagger}{ }^{t}\right\rangle_{-r}\left(g_{0 t}^{\dagger j}\right)$.

\section{Asymptotically free pure gauge models}

As we can see from the pure gauge limit of the original theory, the gauge average is invoked without any weight for the gauge freedom except for the complex phase of the chiral determinant. This way of the gauge average is expected to keep the disordered nature of the gauge freedom. In order to examine the dynamical effect of the gauge average, however, it is desirable to have control over the fluctuation of the gauge freedom [8] by deforming the weight of the gauge average as

$d \mu[g] \rightarrow d \mu[g ; K]=d \mu[g] \exp \left(-K S_{g}[g]\right)$.

For nonabelian gauge groups, as we will argue, this can be acheived without spoiling its disordered nature by introducing an asymptotically free self-coupling of the gauge degrees of freedom.
In two-dimensions, we introduce the nearestneighbor coupling[6]:

$K \sum_{n \mu} \operatorname{Tr}\left(g_{n} g_{n+\hat{\mu}}^{\dagger}+g_{n+\hat{\mu}} g_{n}^{\dagger}\right), \quad\left(K \equiv \frac{1}{\lambda^{2}}\right)$.

In four-dimensions, we consider the covariant gauge fixing term and the Faddeev-Popov determinant[7], following Hata[9] (cf. [10 12]):

$$
-\frac{1}{2 \alpha} \sum_{n}\left(\bar{\nabla}_{\mu} \hat{A}_{n \mu}\right)^{2}-\sum_{n m} \bar{c}_{n}^{a} \hat{M}_{n m}^{a b} c_{m}^{b}\left(\alpha \equiv \lambda^{2}\right),
$$

where

$\hat{A}_{n \mu}=\frac{1}{2 i}\left(U_{n \mu}-U_{n \mu}^{\dagger}\right)-\frac{1}{N} \mathbb{1} \operatorname{Tr} \frac{1}{2 i}\left(U_{n \mu}-U_{n \mu}^{\dagger}\right)$,

with the pure gauge link variable Eq.(2). $\hat{M}_{n m}^{a b}$ is the lattice Faddeev-Popov operator.

We can show by the perturbation theory in $\lambda$ (background field method) that both pure gauge models share the novel features of the twodimensional nonlinear sigma model even in the presense of the imaginary action. The model is renormalizable (at one-loop in four-dimensions) and $\lambda$ is asymptotically free. Severe infrared divergence occurs and it prevents local order parameters from emerging.

Based on these dynamical features, we assume that the global gauge symmetry does not break spontaneously for the entire region of $\lambda$ and the gauge freedom acquires mass $M_{g}$ dynamically through the dimensional transmutation. With this assumption, the decoupling of the gauge freedom could occur as $M_{g} \nearrow \frac{1}{a}$ in the limit $\lambda \nearrow \infty$.

In two-dimensions, an important counter example occurs if the gauge anomaly does not cancel and the actual Wess-Zumino-Witten term appears in the complex action. This term causes an IR fixed point in the beta function of $\lambda \sqrt{13}$. It has been shown to be true also in the vacuum overlap formalism [8]. The fixed point theory is equivalent to the free massless fermion with the chiral $S U(N)$ symmetry 13] and this simply means the failure of the decoupling of the gauge degrees of freedom. It is interesting to note the possibility that the nature of the pure gauge dynamics could distinguish the anomaly-free chiral gauge theory from anomalous ones. 


\section{Boundary correlation function}

The asymptotic freedom allows us to tame the gauge fluctuation by approaching the critical point of the gauge freedom. There we can invoke the spin wave approximation for the calculation of the invariant boundary correlation function

$\left\langle\phi_{n i} \phi_{m}^{\dagger i}\right\rangle_{-r}$,

which is free from the IR divergence. At one-loop order, we obtain

$$
\begin{aligned}
& \frac{1}{2} \delta_{n m} \delta_{i}^{i}-S_{-}^{v}(n-m) \delta_{i}^{i} \\
- & \lambda^{2} \sum_{r} S_{-}^{v}(n-r)\left[\left\langle\pi_{r} \pi_{m}\right\rangle^{\prime} S_{-}^{v}(r-m)\right] \delta_{i}^{i} \\
+ & \lambda^{2} \sum_{r, l} S_{-}^{v}(n-r) \times \\
& {\left[\left\langle\pi_{r} \pi_{l}\right\rangle^{\prime} S_{-}^{v}(r-l)\right] S_{-}^{v}(l-m) \delta_{i}^{i}+\mathcal{O}\left(\lambda^{4}\right), }
\end{aligned}
$$

where

$$
\begin{aligned}
S_{-}^{v}(n-m)= & \int \frac{d^{D} p}{(2 \pi)^{D}} e^{i p(n-m)} \frac{1}{2 \lambda_{-}} \times \\
& \left(\begin{array}{cc}
-m_{0}+B(p) & \sigma_{\mu} \sin p^{\prime} \\
\sigma_{\mu}^{\dagger} \sin p_{\mu} & m_{0}-B
\end{array}\right. \\
B(p)= & \sum_{\mu}\left(1-\cos p_{\mu}\right), \\
\lambda_{-}= & \sqrt{\sum_{\mu} \sin ^{2} p_{\mu}+\left(B(p)-m_{0}\right)^{2}}
\end{aligned}
$$$$
\left(\begin{array}{cc}
-m_{0}+B(p) & \sigma_{\mu} \sin p_{\mu} \\
\sigma_{\mu}^{\dagger} \sin p_{\mu} & m_{0}-B(p)
\end{array}\right),
$$

and

$$
\left\langle\pi_{n} \pi_{m}\right\rangle^{\prime}=\int \frac{d^{D} p}{(2 \pi)^{D}} \frac{e^{i p(n-m)}-1}{\left(\sum_{\mu} 4 \sin ^{2} \frac{p_{\mu}}{2}\right)^{D / 2}} .
$$

It turns out that the spectrum of $S_{-}^{v}$ has the mass gap $M_{B}$ of the order of the cutoff,

$\cosh M_{B}=1+\frac{m_{0}^{2}}{2\left(1-m_{0}\right)}, \quad\left(m_{0}=0.5\right)$.

We also see that the quantum correction due to the gauge fluctuation at one-loop does not affect the leading short-distance nature. There is no symmetry against the spectrum mass gap. Therefore it seems quite reasonable to assume that it holds true as $\lambda$ becomes large. Since the overlap correlation function does not depend on the gauge freedom and does show the chiral spectrum[1], the above fact means that the entire fermion spectrum is chiral.

The author would like to thank H. Neuberger and R. Narayanan for enlightening discussions. He also would like to thank H. Hata, S. Aoki, H. So and A. Yamada for discussions.

\section{REFERENCES}

1. R. Narayanan and H. Neuberger, Nucl. Phys. B412 (1994) 574; Phys. Rev. Lett. 71 (1993) 3251; Nucl. Phys. B(Proc. Suppl.)34 (1994) 95,587; Nucl. Phys. B443 (1995) 305.

2. D. Foerster, H.B. Nielsen and M. Ninomiya, Phys. Lett. B94 (1980) 135; S. Aoki, Phys. Rev. Lett. 60 (1988) 2109; Phys. Rev. D38 (1988) 618; K. Funakubo and T. Kashiwa, 60 (1988) 2113; J. Smit, Nucl. Phys. B(Proc. Suppl.)4 (1988) 451.

3. D.B. Kaplan, Nucl. Phys. B30(Proc. Suppl.) (1993) 597.

4. M. Golterman, K. Jansen, D. Petcher and J. Vink, Phys. Rev. D49 (1994) 1606; M.F.L. Golterman and Y. Shamir, Phys. Rev. D51 (1995) 3026.

5. M.F.L. Golterman and Y. Shamir, Phys. Lett. B353 (1995) 84; Erratum-ibid. B359 (1995) 422. R. Narayanan and H. Neuberger, Phys. Lett. B358 (1995) 303.

6. Y. Kikukawa, KUNS-1445 HE(TH)97/08, May 1997, hep-lat/9705024.

7. Y. Kikukawa, KUNS-1446 HE(TH)97/09, July 1997, hep-lat/9707010.

8. Y. Kikukawa and S. Miyazaki, Prog. Theor. Phys. 96 (1996) 1189.

9. H. Hata, Phys. Lett. 143B (1984) 171.

10. A. Borrelli, L. Maiani, G.C. Rossi, R. Sisto and M. Testa, Nucl. Phys. B333 (1990) 335.

11. Y. Shamir, TAUP-2306-95, Dec 1995; Y. Shamir, Nucl. Phys. B(Proc. Suppl.)53 (1997) 664.

12. M.F.L. Golterman and Y. Shamir, Phys. Lett. B399 (1997) 148.

13. E. Witten, Commun. Math. Phys. 92 (1984) 455 . 